【国際セッション・オーラルプレゼンテーション】

\title{
Apoptotic development in pathological muscle damage in a time course study of pressure-induced deep tissue injury
}

Teng BT, Tam EW, Benzie IF, Siu PM

Department of Health Technology and Informatics, the Hong Kong Polytechnic University, Hong Kong

Deep pressure ulcer still exists as a significant health problem owing to the limited understanding of clinical etiologies that confines the current diagnosis and prevention of deep tissue injury. Muscle tissue as the origin of tissue ulceration is of greater clinical concern nowadays due to the greater vulnerability of muscle tissue to sustained mechanical loading resulting in a higher level of pressure and shear at muscle-bone interface. We have recently demonstrated that apoptosis is activated in the presence of degenerative morphological damage in muscle tissue in an adopted animal model of deep tissue injury. This suggests that muscle apoptosis plays a destructive role in the underlying mechanism of pressure-induced deep tissue injury. Nonetheless, the exact onset of this activation causing muscle damage is still not known. Our study aims to investigate the sequential cellular breakdown event in compression-induced muscle pathology using an in vivo animal model. Adult Sprague Dawley rats were subjected to an in vivo pressure ulcer protocol as induced by moderate and cumulative prolonged compression. Static pressure of $100 \mathrm{mmHg}$ was applied to an area of $1.5 \mathrm{~cm}^{2}$ over the tibialis region of the right limb of the rats (i) for 1 session of 3 hours loading (3h), (ii) 1 session of 6 hours loading $(6 \mathrm{~h})$, and (iii) 1 session of 6 hours loading each day for 2 consecutive days $(6+6 \mathrm{~h})$. The left unloaded limb served as intra-animal control. Muscle tissue underneath the compression region was collected from all groups. The morphological characteristic of muscle tissues was demonstrated histologically by hematoxylin and eosin staining, whereas the protease activity of caspase -3 and -8 was analysed using a fluorometric assay. Apoptotic DNA fragmentation was assessed by cell death enzyme-linked immunosorbent assay (ELISA). We found no observable morphological change in the compressed muscle fibers of $3 \mathrm{~h}$ and $6 \mathrm{~h}$ groups, yet nuclei started to aggregate in the interstitial space of $6 \mathrm{~h}$ muscle group with the integrity of muscle cells was still retained. Degenerative characteristics, which include the rounding of myofibers and nuclei accumulation, were observed in $6+6 \mathrm{~h}$ group. Our quantitative DNA fragmentation analysis demonstrated there was a significant elevation of the apoptotic DNA fragmentation for about 10.6 and 9.8 folds in the compressed muscle of $6 \mathrm{~h}$ and $6+6 \mathrm{~h}$ respec tively relative to the control muscle $(\mathrm{P}<0.05)$. The activation of caspase- 3 was confirmed in cytosolic protein extracts in which there was about 2.2 and 2.9 folds increase in the caspase- 3 protease activity in compressed muscle of $6 \mathrm{~h}$ and $6+6 \mathrm{~h}$ groups respectively compared to the control $(\mathrm{P}<0.05)$. There was also approximately 1.6 folds elevation of cytosolic caspase- 8 activity in 6 h group of compressed muscle $(P<0.05)$. As a whole, our study intends to provide a better understanding on the sequential events in terms of its underlying biochemical changes governing the apoptotic development in the initiation and progression of the compression-induced muscle pathology. This idea may provide a critical influence on future treatments plans as therapeutic target in alleviating the pathological muscle damage as a result of moderate compression in pressure-induced deep tissue injury . 